ARTICLE OPEN

\title{
Enhancement of orbital ordering and spin polarization by controlling the dimensionality of the octahedra network
}

\author{
Mingqiang $\mathrm{Gu}^{1,2}$, Kang Wang ${ }^{1}$, Yiming Wang ${ }^{1}$, Qiyun Xie ${ }^{1}$, Hongling Cai ${ }^{1}$, Guo-Ping Zhang ${ }^{3}$ and Xiaoshan Wu ${ }^{1}$
}

Two-dimensional (2D) systems have been widely investigated with perovskite superlattices because they show excellent epitaxy. A challenging question is naturally raised: whether lower dimensionality, e.g., one dimension (1D) and zero dimension (0D), can be achieved by perovskites? In this work, we propose a way to control the dimensionality of the octahedra network in perovskite superlattices by selecting the substrate orientation and superlattice period. Taking $\mathrm{SrTiO}_{3} / \mathrm{SrRuO}_{3}$ as an example, we demonstrate that the 1D structure is in a 1D Ising state, which is paramagnetic, while the OD structure is ferromagnetic insulator with fully saturated magnetic moment on the Ru sites. New phenomena in the magnetic and electronic properties are observed, including large strain response, half-metallicity, and orbital-selective quantum confinement effects.

npj Quantum Materials (2016) 1, 16011; doi:10.1038/npjquantmats.2016.11; published online 16 September 2016

\section{INTRODUCTION}

Heterojunctions of two perovskites can be now fabricated in an atomically flat fashion with the advent of the state-of-the-art pulse laser deposition. It provides an effective artifice to confine the electron motion within two dimension. Numerous remarkable properties have been discovered in these systems, e.g., twodimensional electron gas, multiferroics, the coexistence of ferromagnetism and the superconductivity, etc. ${ }^{1,2}$ making it a sustaining attractive topic in the last decade. One representative example is the $\mathrm{SrRuO}_{3} / \mathrm{SrTiO}_{3}$ (SRO/STO) hybrid system. A twodimensional (2D) half-metallic state can be built by simply embedding a single layer of metallic SRO into the insulating $\mathrm{STO}^{3}$ Moreover, the magnitude of Ru magnetic moment in such superlattice has been proved tunable according to the applied strain. ${ }^{4,5}$ Manipulating the electronic and magnetic properties in this system sheds lights on the future design of functional devices.

Although most of the previous studies investigated thin films grown on conventional (001)-oriented substrates, recent works have shown that thin film properties can be improved by applying different substrate orientations. ${ }^{6,7}$ The superexchange interaction strength of $\mathrm{BiFeO}_{3}$ can be weakened by using a (111)-oriented substrate. ${ }^{6}$ Also, the octahedra tilt pattern for $\mathrm{NdNiO}_{3}$ can be changed by growth on a (111)-oriented $\mathrm{LaAlO}_{3}$ substrate, which leads to a noncentrosymmetric metallic phase. ${ }^{7}$ Therefore, choosing the correct substrate orientation is one of the key issues in material design. Here we report that by using substrates with different crystallographic orientations, one can control the dimensionality of the connection of the octahedra network in the superlattice (shown in Figure 1). These systems have distinct advantages compared with the nano systems. (i) They are well embedded in the bulk materials and therefore have nice contact. (ii) The B-site ion is selective by changing the mother compound to get a certain required property, e.g., proper exchange or correlation strength between sites, which provides flexibility for novelty.

Traditionally, dimensionality lower than 2D, i.e., one-dimension (1D) or zero dimension (OD), is achieved with the nano fabrication of nanowires and nanotubes (1D), or nano clusters like $C_{60}(0 D)$ and quantum dots. Most of these structures are dissociative units and difficult to have an excellent contact with another material like an electrode, which limits their application. On the other hand, several nanostructures, e.g., nano lines, have been observed by scanning tunneling microscopy on STO (001) and (110) surfaces., ${ }^{8,9}$ These nanostructures provide a hint that perovskites have inherent advantage to be the base of low dimensional systems. How to build a lower dimensional system that manifests distinct macroscopic properties is a challenge.

In the bulk $\mathrm{ABO}_{3}$ perovskites, the $\mathrm{BO}_{6}$ octahedra form a threedimensional network spanning throughout the space. The dimensionality of the $\mathrm{BO}_{6}$ octahedra network can be changed by forming interface with a superlattice construction. When the $\mathrm{ABO}_{3} /$ $A^{\prime} B^{\prime} O_{3}$ type superlattice is grown on a conventional (001)-oriented substrate, the $\mathrm{BO}_{6}$ octahedra form a two-dimensional network, see Figure 1a (here we assume the superlattice periodicity is one by one). Each $\mathrm{BO}_{6}$ octahedron connects with four $\mathrm{BO}_{6}$ octahedra by sharing the oxygens at the corners in the $x-y$ plane. Along the $z$ direction, i.e., the growing direction, the connection is truncated by the upper and lower $\mathrm{B}^{\prime} \mathrm{O}_{6}$ octahedra. By selecting proper $\mathrm{B}$ and $\mathrm{B}^{\prime}$ element, $2 \mathrm{D}$ electron gas is formed in structures of this type.,10 When the (110)-oriented substrate is used, the growing direction $z$ deviates by $45^{\circ}$ from the local $z$ axis of the octahedron coordinates. Each $\mathrm{BO}_{6}$ octahedron connects with two $\mathrm{BO}_{6}$ octahedra along one of the in-plane axes, for example the $y$ axis in Figure 1(b), which connects octahedra arrays parallel to the $y$ axis. Recent study has observed large anisotropy in the transport property of the 2D electron gas in the (110)-oriented $\mathrm{LaAlO}_{3} / \mathrm{SrTiO}_{3}$ system, ${ }^{11,12}$ and may be explained by the low-dimensional octahedral network. Finally on the (111)-oriented substrate, the superlattice has a OD

\footnotetext{
${ }^{1}$ Nanjing National Laboratory of Microstructures, Laboratory of Solid State Microstructures, Photovoltaic Engineering Center, School of Physics, Nanjing University, Nanjing, China; ${ }^{2}$ Department of Materials Science and Engineering, Northwestern University, Evanston, IL, USA and ${ }^{3}$ Department of Physics, Indiana State University, Terre Haute, IN, USA. Correspondence: XS Wu (xswu@nju.edu.cn)

Received 17 April 2016; revised 30 July 2016; accepted 1 August 2016
} 
Octahedral network:

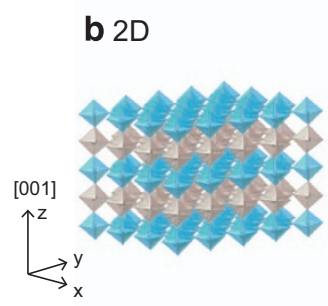

a Bulk perovskite I

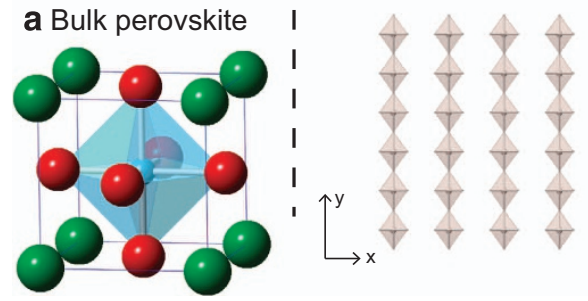

d $O D$ and derived $O D$

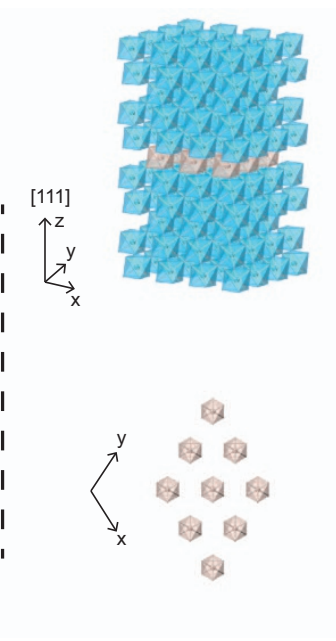

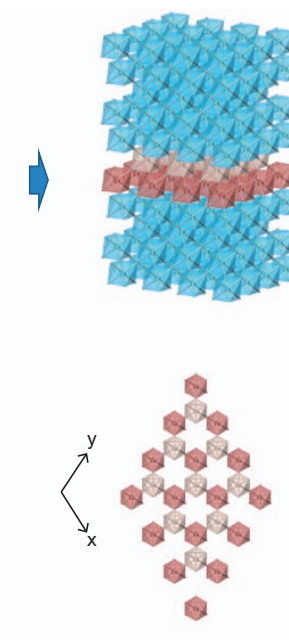
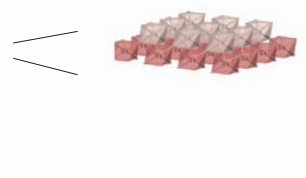

e connection mode in $\mathrm{OD}$

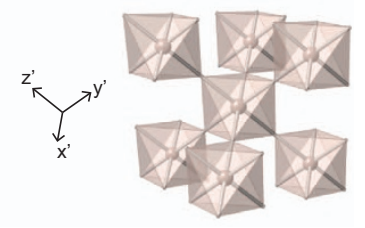

Figure 1. Dimensionality of the octahedra network in perovskite supperlattices. (a) Structure of a generic perovskite crystal. (b) Twodimensional (2D) network built by embedding a single layer into the (001)-oriented substrate. (c) One-dimensional (1D) structure of the octahedra network built by using substrate with (110) orientations. The upper and lower patterns are views of the 1D structure on the whole and extracted, respectively. (d) Zero-dimensional (OD) and derived OD structures in the supperlattices with (111)-oriented substrates. The upper and lower patterns are views of the $\mathrm{OD}$ and derived $\mathrm{OD}$ structures on the whole and extracted, respectively. (e) Connection mode in $0 \mathrm{D}$ networks. The six nearest neighboring octahedra of the $\mathrm{BO}_{6}$ are all $\mathrm{B}^{\prime} \mathrm{O}_{6}$, and vice versa.

structure (Figure 1(c)): The six nearest neighboring octahedra of the $\mathrm{BO}_{6}$ are all $\mathrm{B}^{\prime} \mathrm{O}_{6}$, and vice visa. Therefore, if we stick with the $\mathrm{BO}_{6}$ network itself, we will find that all the $\mathrm{BO}_{6}$ octahedra are isolated.

The thickness of the two parent perovskites within the superlattice period is another parameter to tune the connection type of the octahedra network. Take the (111)-oriented superlattice for example, if there is only one $\mathrm{ABO}_{3}$ layer within one period, i.e., a $\left[\mathrm{ABO}_{3}\right]_{1} /\left[\mathrm{A}^{\prime} \mathrm{B}^{\prime} \mathrm{O}_{3}\right]_{n}$ super-lattice, the $\mathrm{BO}_{6}$ network is in $O D$, as mentioned before. If there are two or more consecutive $\mathrm{ABO}_{3}$ layers within one period, i.e., a $\left[\mathrm{ABO}_{3}\right]_{m} /\left[\mathrm{A}^{\prime} \mathrm{B}^{\prime} \mathrm{O}_{3}\right]_{n}$ superlattice with the integer $m>1$, the $\mathrm{BO}_{6}$ octahedra are connected with one another in a zig-zag way. We call this a 'derived OD' structure. Such a special connection way affects the hybridization between the $\mathrm{O}^{2-}$ and the $\mathrm{B}^{\prime}$ site ions and thus the exchange coupling strength between neighboring cations.

\section{RESULTS}

In this work, we select SRO and STO as the two parent materials to build the superlattices, with the idea that the relative extended $\mathrm{Ru}$ $4 d$ states are truncated by the localized Ti $3 d$ states. ${ }^{3}$ In this sense, we realize for the first time a systematic manipulation of the $\mathrm{Ru} 4 d$ electron from an extended 2D state to a confined 1D chain state, and finally down to a localized OD state. The magnetic moment on the Ru site is enhanced and controllable. A sharp ferromagneticnonmagnetic phase transition is noticed theoretically, which is an evidence that the magnetic property in this system is extremely sensitive to strain. Also, orbital selectivity is noticed among the three $\mathrm{Ru}-\mathrm{t}_{2 g}$ orbitals.

In the SRO/STO system, pioneer efforts have shown that SRO thin films growing on the STO substrate with the orientation other than (001) lead to nontrivial electronic states. Experimentally, the magnetic moment was observed to exceed the low-spin limit of $2 \mu_{\mathrm{B}}$ per Ru atom in the SRO thin film grown on (111) oriented substrate. $^{13}$ There is, however, still no evidence to substantiate whether this observation is intrinsic to the system or an extrinsic effect. Theoretical investigation with first-principles calculations found that the spin direction of the surface top layer collapsed from out-of-plane to in-plane when the film thickness was below two atomic layers. ${ }^{14}$
The electronic ground state of SRO is well known as an itinerant ferromagnet, which is well characterized by the Stoner model. ${ }^{15}$ Taking into account the large crystal field splitting between $\mathrm{e}_{g}$ and $t_{2 g}$ states, the Hund's rule predicts the magnetic moment for each $\mathrm{Ru}^{4+}$ site to be fully saturated as $2 \mu_{\mathrm{B}}$ in the ionic limit $\left(t_{2 g}^{3} \uparrow, t_{2 g}^{1} \downarrow\right)$. Experimentally, however, the measured magnetic moment in this system typically ranges from 0.8 to $1.6 \mu_{B} / R u$, rather than fully saturated. This can be attributed to the enhanced spin fluctuations due to the electron itineracy. This means that if one can suppress the metallicity, the magnetic moment will increase. In this sense, the $\mathrm{OD}$ configuration is an ideal structure to serve this goal as it isolates each of the $\mathrm{RuO}_{6}$ octahedra, making the electrons difficult to hop between $\mathrm{Ru} 4 d$ and $\mathrm{t}_{2 g}$ orbitals. On the other hand, the electron hopping is confined within the octahedra chain along the $y$ axis for the 1D superlattice, while difficult to happen along the other axes. This leads to a unique hybridization situation between $\mathrm{Ru}$ and the surrounding oxygens, which provides an opportunity to study the 1D metallic Ising chain.

[SRO]/[STO] superlattice samples are grown on (001)-, (110)- and (111)-oriented STO substrates using pulse laser deposition method. All the samples have a superlattice period of 1:1. The quality of the epitaxial film is strictly controlled in experiments, as that described in our previous work. The $\mathrm{M}-\mathrm{T}$ and $\mathrm{M}-\mathrm{H}$ hysteresis loops are measured using Superconducting Quantum Interference Device (SQUID), plotted in Figure 2. As it is known, the finite temperature solution for the 1D Ising model is a paramagnetic state, which is in agreement with our measurement. However, although small, there is non-vanishing magnetic moment in our 1D sample. This is owing to the extended character of the $4 d$ electrons. There is interaction between neighboring $\mathrm{RuO}_{6}$ chains, which draws the system back from truly 1D. The system is close to insulating and therefore feasible to be described by the Heisenberg model. In this case, the system should hold a fully spin-polarized state, i.e., $2 \mu_{B} / R u$. The measured magnetic moment has an increase compared with the $2 \mathrm{D}$ case, however, it is still smaller than the predicted value. This may also be owing to the extended feature of the $4 d$ electron, or the disorder in our sample during thin film fabrication.

To get insight into the current results, we performed further investigation systematically with the $O D$ superlattice. Samples of $[\mathrm{SRO}]_{\mathrm{m}} /[\mathrm{STO}]_{\mathrm{n}}$ with different superlattice periods ( $m: n=1: 1,1: 2,2: 1$, 

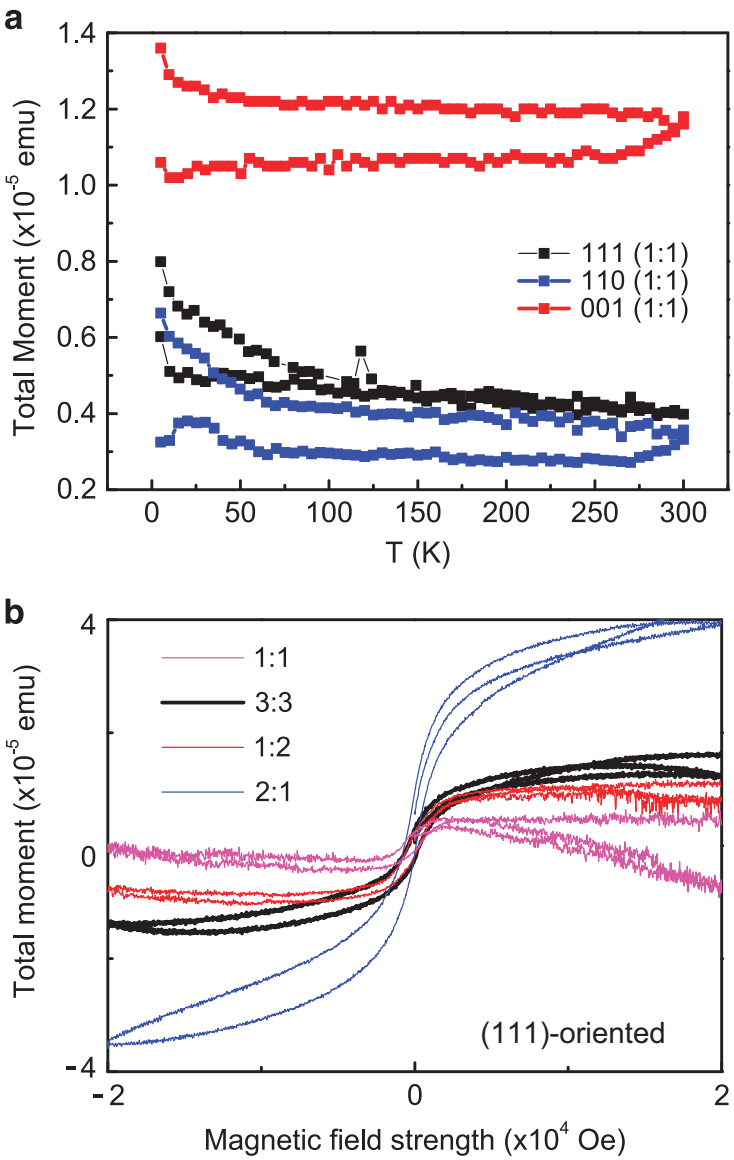

Figure 2. Magnetic measurements of the two-dimensional (2D), onedimensional (1D), and various zero-dimensional (OD) samples. (a) Magnetic moment as a function of temperature for the 2D, 1D, and OD samples, all the samples have the superlattice period of 1:1. (b) $\mathrm{M}-\mathrm{H}$ hysteresis loops for the $\mathrm{OD}$ samples with different supperlattice periods. Measurements are performed at $5 \mathrm{~K}$.

and $3: 3$ ) on the (111) substrate are prepared to clarify the observed phenomena. To have theoretical contrast, density functional theory simulation is also performed. The magnetic moments of these superlattices are shown in Figure 2b. The results in experiment and simulation show good consistency, both revealing obvious increase in the magnetic moment. Especially for the 1:2 and 2:1 case, density functional theory shows that the magnetic moment in the supercell is close to saturation, although the magnetic moment for the 1:2 superlattice shows considerable discrepancy from the theoretical value in our experiments. This is probably owing to the imperfection in the sample: Along the growing direction of (111)-oriented thin film, there are two consecutive charged atomic planes, $\left(\mathrm{SrO}_{3}\right)^{4-}$ and $\mathrm{Ti}^{4+} / \mathrm{Ru}^{4+}$ planes, respectively. Therefore, a certain extent of intermixing at the interface during the growth of superlattice should be expected, which could lead to a departure in the results.

The Ru magnetic moment varies as the superlattice period changes. The matched results in experiment and theory grant us the opportunity to explore the evolution of magnetic moment in this system. First of all, the 1:1 superlattice shows unexpected small moment. This can be caused by the small but non-vanishing coupling between $\mathrm{Ru}$ ions. We check this by theoretically increasing the thickness of STO layer. The 1:2, 1:5 and 1:11 superlattices all represent a near saturated moment. The special $1: 1$ case out of all other $O D$ superlattices shows that owing to the competition between the Ru distance and the vanishing length of the Ru- $4 d$ electrons, the coupling in this system is subtle. There are other ways to check the moment versus $\mathrm{Ru}-\mathrm{Ru}$ distance. For example, one can double the $x-y$ scale of the supercell, while keep only one $\mathrm{Ru}$ atom in the supercell. Tensile strain is also a decent candidate to manipulate the Ru-Ru distance. These will be discussed in the next section.

The magnetic moment from $O D$ to derived $O D$ varies with respect to the superlattice period. The values for 1:1, 2:1 and 3:3 are $0.02,1.75$ and $0.87 \mu_{\mathrm{B}} / \mathrm{Ru}$, respectively. The moment in the 2:1 case increases, even exceeds the bulk value of $\sim 1.03 \mu_{B} / R u$. This qualitatively agrees with the previous experimental results that the (111)-oriented SRO thin films possess a larger magnetic moment. We examine the magnetic moment of each $\mathrm{Ru}$ ion (integrated within the Wigner-Seitz radius of $1 \AA$ ) in the 2:1 and 3:3 structures (denoted in Figure 3a). For the 2:1 case, both Ru ions are located at the interface, with the magnetic moment of $1.2 \mu_{\mathrm{B}}$. For the 3:3 case, there are two interfacial Ru ions and one at the center. The magnetic moment of the interfacial $\mathrm{Ru}$ is $0.76 \mu_{\mathrm{B}}$, while that of the center one is $0.18 \mu_{\mathrm{B}}$. From these results, one finds that the (111) interface has different impacts on the interfacial $\mathrm{Ru}$ moment and the internal ones. The magnetic coupling between $\mathrm{Ru}$ atoms in this system is complicated.

To see the interface effect in detail, we change the thickness of the SRO layer while keeping that of STO layer fixed. Results in Figure $3 \mathrm{~b}$ clearly shows that the average magnetic moment drops when $m$ is larger than 2. If we look into the magnetic moment projected onto each $\mathrm{Ru}$ ion at different layers, the vibration feature is observed in Figure 3c. The largest magnetic moment is always found at the interface. For $m=1$ and $m=2$ structures, the interfacial moment is similar, $\sim 1.2 \mu_{\mathrm{B}}$. For $m>2$, the interfacial moment slightly drops to $\sim 1 \mu_{\mathrm{B}}$, whereas that for the internal Ru atoms ranges from $0.14 \mu_{\mathrm{B}}$ to $0.88 \mu_{\mathrm{B}}$. The $\mathrm{Ru}$ atom adjacent to the interfacial one generally undergoes suppression in the magnetic moment. As the SRO thickness increases, the internal Ru moment gradually returns to the bulk value at the fifth layer off the interface.

For the ultrashort 1:1 superlattice, the magnetic moment is unexpectedly suppressed. Theoretically, the magnetic property of this structure is subtle. When the structure has perfect inversion symmetry, the magnetic moment is quenched; when the symmetry is artificially broken by slightly displacing the atoms in the $z$ direction, a small but noticeable magnetic moment of $\sim 0.11 \mu_{\mathrm{B}} / \mathrm{Ru}$ is found. As in practice, perfect inversion symmetry is broken by the thin film-substrate interface and the surface, the quenched-spin state is usually concealed. Therefore, a small magnetic moment should be expected in this structure.

As is discussed before, both the measured and theoretical magnetic moment for the Ru chains in the (110)-oriented thin film is not strictly zero which is predicted for ideal 1D Ising chain. A residue moment of $0.03 \mu_{\mathrm{B}}$ is found on the $\mathrm{Ru}$ atom. We attribute this to the non-vanishing coupling between neighboring $\mathrm{RuO}_{6}$ chains owing to the relatively extended feature of the Ru-4d electrons. To verify this guess, the relationship between Ru-moment and $\mathrm{Ru}-\mathrm{Ru}$ distance should be examined. The supercell method is used. The cell dimension along $a$ is doubled, and one of the two Ru atoms is replaced by $\mathrm{Ti}$ atom (i.e., one $\mathrm{Ru}$ and three $\mathrm{Ti}$ atoms are contained in the supercell). In this way, the distance between neighboring $\mathrm{RuO}_{6}$ chains is doubled. The calculated $\mathrm{Ru}$ moment in this structure is zero. It vindicates our assumption that the residue moment originates from the coupling between the $\mathrm{RuO}_{6}$ chains owing to the extended Ru electrons. Similarly, we find that the nonsaturated moment for the $\mathrm{OD}(1: 2)$ structure is also attributed to the extended feature of the extended feature of the Ru electrons. When the distance of Ru atoms is doubled by expanding the unit cell, the fully saturated magnetic moment of $2 \mu_{B}$ is found. Note that the onsite Coulomb correlation is turned off in our calculation. Thus such fully saturated state indicates that the $\mathrm{Ru}^{4+}$ ion achieves the upperlimit of the low-spin state $\left(t_{2 g}^{3} \uparrow, t_{2 g}^{1} \downarrow\right)$. The system is turned from an 

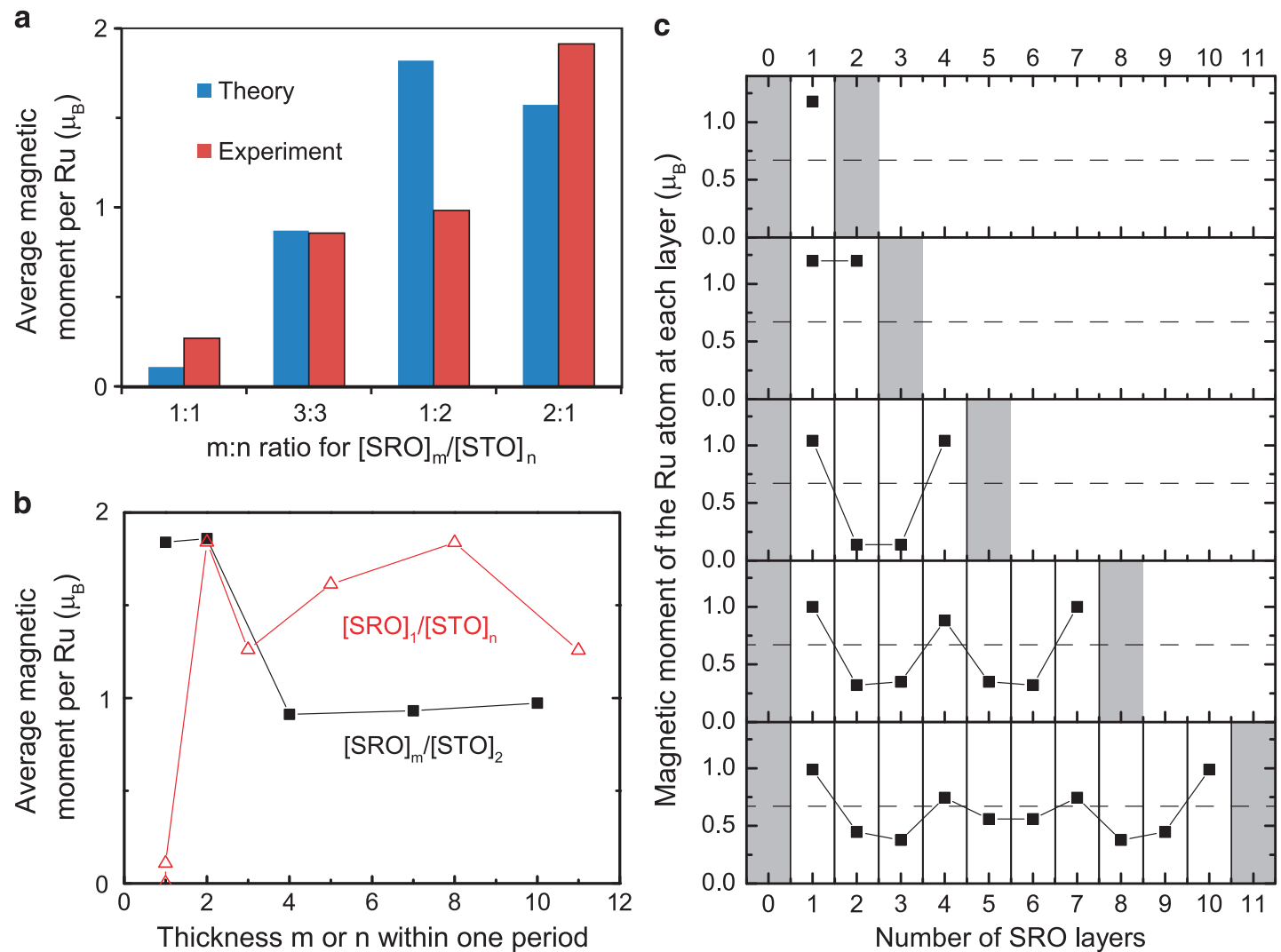

Figure 3. Magnetic properties of zero-dimensional (OD) and derived OD networks. (a) Average magnetic moment each Ru owns for samples of $[\mathrm{SRO}]_{m} /[\mathrm{STO}]_{n}$ with different supperlattice periods ( $m: n=1: 1,1: 2,2: 1$ and 3:3) on the (111)-oriented substrates. The red bars represent experimental results and blue bars represent theory results. (b) Density functional theory (DFT) simulations of magnetic moments in supperlattices with OD (red curve) and derived OD (black curve) networks. (c) Evolution of magnetic property from OD to derived OD networks by increasing the thickness of SRO layers with fixing that of STO layers. It shows magnetic moment that each SRO layer owns.

itinerant ferromagnetic state into the localized ferromagnetic state, maximizing the magnetic moment.

Experimentally, however, it is not practical to double the unit cell size and form such regular supercell structures. One effective way to change the distance between neighboring $\mathrm{RuO}_{6}$ chains (or $\mathrm{RuO}_{6}$ octahedra) in the 1D (OD) structure is to apply the epitaxial strain. Therefore, we investigate the strain effects. We artificially change the in-plane lattice constant of the structure and check the evolution of the magnetic moment. The results are plotted in Figure 4. It is clear that when the Ru-Ru distance increases, the magnetic moment in the 1D structure drops to zero, whereas that in the OD structure increases to fully saturate. Note that in the strained cases, not only the distance between neighboring $\mathrm{Ru}$ atoms is changed, but also the local environment within the octahedron (i.e., the $\mathrm{Ru}-\mathrm{O}$ bond lengths) is also changed. Therefore the strain effect has combined contributions from these two effects. The overall trends are in agreement with the supercell method, confirming that our theory works well in these systems. On the other hand, the magnetic moment for the OD structure in Figure 4 drops sharply under a compressive strain, which is consistent with previous results found by Zayak et al. ${ }^{5}$ in bulk SRO. In our case, the change is rather significant, from a nearly fully saturated value, $\sim 1.80 \mu_{\mathrm{B}}$, to zero, which suggests the potential application of the $0 \mathrm{D}$ structure.

\section{DISCUSSION}

We examine the density of states (DOS) to check the spin polarization. Figure 5 shows the DOS projected on the Ru-4d

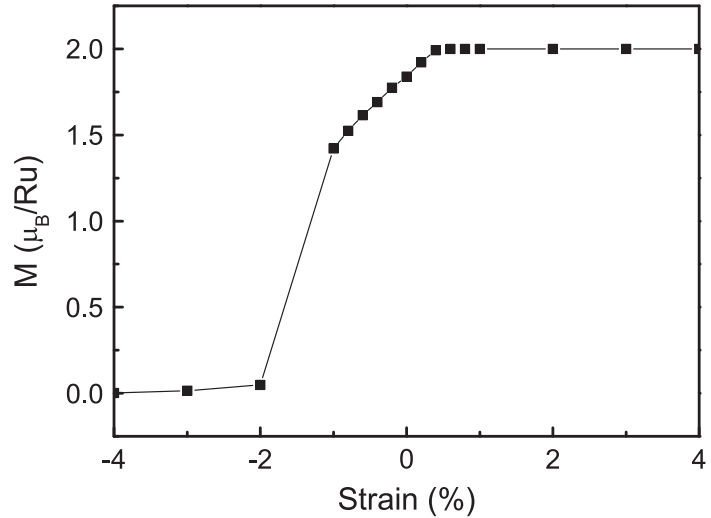

Figure 4. Magnetic moment for the Ru chain with strain effect. The Ru chain is built in the (110)-oriented thin films. By modulating lattice strain, magnetic moment changes because of the variations of coupling between neighboring $\mathrm{RuO}_{6}$ chains.

orbitals (pDOS) for different structures. Owing to crystal field splitting and the four-electron occupation condition, the Ru- $t_{2 g}$ orbitals dominate the physics near the Fermi level. The most interesting result is that for the $O D$ superlattice, shown in Figure $5 \mathrm{a}$. The spin-up channel of the $t_{2 g}$ orbitals are nearly fully occupied, whereas the spin-down orbitals are occupied by the remaining electrons in an orbital-selective way. To check the 


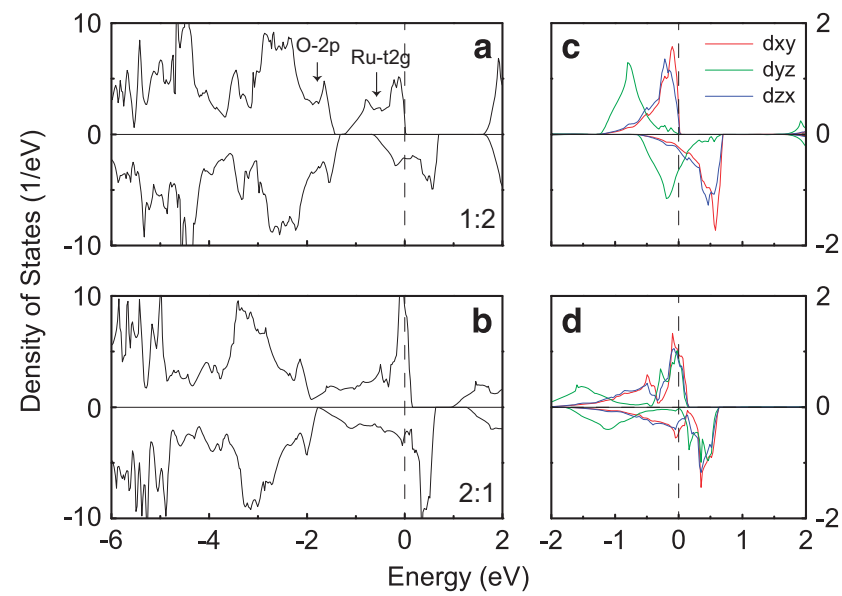

Figure 5. Density of states (DOS) projected on the Ru-4d orbitals (pDOS) for different structures. (a) pDOS for the OD supperlattice. (b) pDOS for the derived OD supperlattice. (c) Partial DOS of $d_{x y, y z, z x}$ orbitals for the OD supperlattice. (d) Partial DOS of $d_{x y, y z, z x}$ orbitals for the derived OD supperlattice. The projection to the $t_{2 g}$ manifold is according to the local coordination, i.e., along the direction of the $\mathrm{Ti}-\mathrm{O}$ bonds. The positive and negative values represent spin-up and spin-down channels, respectively.

orbital occupation in detail, we integrate the DOS from $-2.2 \mathrm{eV}$ to the Fermi level to get the number of electrons. We find that $\sim 0.6$ electrons occupy the $d_{y z}$ orbitals, and only $\sim 0.2$ electrons reside in the $d_{x y}$ and $d_{z x}$ orbitals. The orbital-selective electron occupation leads to weak orbital ordering for the three spin-down orbitals. Compared with that theoretically found in the bulk SRO, the lifted orbital degeneracy in our $\mathrm{OD}$ system is much robust, as it exists even without any on-site Coulomb correction. ${ }^{16}$ Also, this feature survives when the electron correlation effect and spin-orbit coupling are taken into account. The magnetic moment for the $\mathrm{Ru}$ ion is increased upon this degeneracy lifting.

Such orbital-selective electron occupation is caused by the quantum confinement effect in $0 \mathrm{D}$ system. As indicated from the structure analysis, in the $\mathrm{OD}$ structure, the $\mathrm{RuO}_{6}$ octahedra are isolated by $\mathrm{TiO}_{6}$. The localized feature can be noticed in the partial DOS plotted in Figure 5c: Three localized peaks correspond to the van-Hove singularity of the truncated $d_{x y, y z, z x}$ orbitals, respectively. In the derived $O D$ case $(2: 1)$, as the thickness of the SRO layer increases, the three localized peaks still exist. The band width of these bands is broadened and hybridized with the O-2p bands. As the number of $4 d$ electrons increases, three extra sharp peaks emerge at higher energy and the Fermi level is lifted upward. In this way, the orbital selective occupation feature for spin-up channel disappears.

We experimentally measured the transport properties of our samples. The comparison between the $O D$ and the derived $O D$ is interesting. The temperature dependence of the resistivity clearly shows that the $O D$ structure is insulating within the whole measured temperature region. On the other hand, at high temperature, the derived $O D$ structure behaves as a metal, and when $T<T_{\mathrm{c}} \approx 88 \mathrm{~K}$, this structure undergoes a metal-to-insulator transition. This experimental result seems to contradict the theoretical calculation, which always show non-vanishing DOS at the Fermi level. Though this inconsistency is usually the case for the SRO thin film system, ${ }^{14,15,17}$ one may resort to the semiempirical correction of the exchange correlation to the LDA model, e.g., LDA $+U .{ }^{16}$ This may work; however, its result depends on a tunable parameter $U$ and loses the generic meaning. In this work, we use the hybrid functionals (HSE06) in the calculation, to include the more precise electron-electron correlation selfconsistently. After including this correction, the theoretical and

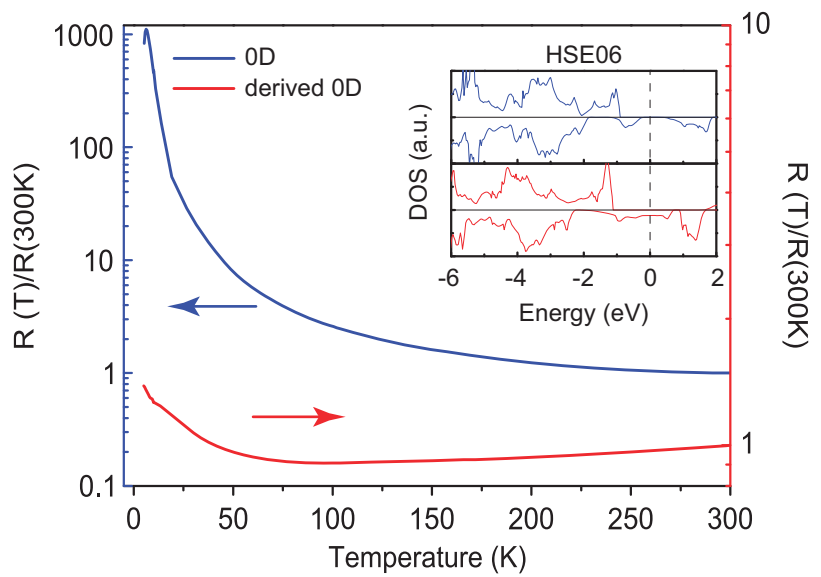

Figure 6. Transport properties of zero-dimensional (OD) and derived OD networks. The temperature dependence of the resistivity for OD (blue curve) and derived OD (red curve) structures. The inset is the density of states (DOS), using the HSEO6 in the calculation with a high-effective on-site Hubbard correlation $U_{\text {eff }}=4 \mathrm{eV}$.

experimental results show excellent agreement: A band gap is opened in the DOS of OD structure, while the derived OD structure is still metallic, shown in Figure 6.

Finally, comparison between our theoretical and experimental results indicates that when considering the transport property of the SRO/STO system, the electron correlation should be taken high effective on-site Hubbard correlation $\left(U_{\text {eff }}=4 \mathrm{eV}\right)$. On the other hand, when considering the magnetic property, this contribution should be excluded to reflect the correct trend of magnetic moment change. This can be a clue to solve the debate on the contradicting coercive field between magnetic hysteresis measurement and transport measurement, which is beyond the scope of this study.

In summary, we have theoretically designed and experimentally achieved the control of magnetic properties for the SRO/STO hybrid system. The magnetization is suppressed in the 1D structure, consistent with a 1D Ising chain model. Enhancement of Ru magnetic moment is observed at the interface of the $O D$ and derived $\mathrm{OD}$ structures. An interesting orbital-selective quantum confinement effect is observed in the OD structure, which results in lifting of the $\mathrm{Ru} t_{2 g}$ orbitals, localizing these states below Fermi level. In all, the magnetic and electronic properties are subtle because the $\mathrm{Ru}-\mathrm{Ru}$ distance is controlled to a comparable range to the vanishing length of $\mathrm{Ru} 4 d$ electron.

\section{MATERIALS AND METHODS}

$A b$ initio calculations and analysis

We perform first-principles density functional theory calculations within the local density approximation plus on-site Coulomb interaction (LDA+U) as implemented in the Vienna $A b$ Initio Simulation Package ${ }^{18}$ with the projector augmented wave ${ }^{19}$ method to treat the core and valence electrons with the following electronic configurations: $3 s^{2} 3 p^{6} 4 s^{2}$ for $\mathrm{Sr}$, $4 p^{6} 5 s^{2} 4 d^{6}$ for $\mathrm{Ru}$, and $2 s^{2} 2 p^{4}$ for $\mathrm{O}$. We use a plane-wave cutoff energy of $500 \mathrm{eV}$ and the $\Gamma$-centered Monkhost-Pack $k$-point mesh to converge the total energy within $0.01 \mathrm{eV}$. Correlation effect of Ru- $4 d$ electrons is taken into account with an effective $U_{\text {eff }}=2.1 \mathrm{eV}$ within the Dudarev scheme. ${ }^{20}$ The value of $U_{\text {eff }}$ is consistent with the previous studies, ${ }^{4,17}$ giving a reliable description to this system. To exclude the ambiguity brought by the selection of $U$ and have more accurate results, we also perform the HSEO6 type hybrid functionals ${ }^{21}$ calculations. As tested in the previous study, ${ }^{3}$ the hybrid functionals calculation predicts a correct itinerant ferromagnetic ground state for SRO. In the current study, it shows the best consistency to the transport measurement, as shown in Figure 5. 


\section{Experimental setups}

For the growth of OD and derived OD SRO system, [SRO]/[STO] supperlattice samples are grown on (111)-oriented STO substrates using laser-MBE at $750{ }^{\circ} \mathrm{C}$, with the repetition frequency $(2 \mathrm{~Hz})$ and the constant laser intensity of $60 \mathrm{~mJ} / 3.4 \mathrm{~mm}^{2}$. All the samples were grown under an oxygen pressure of $0.005 \mathrm{mbar}$. The growth of supperlattice films was monitored by reflection high energy electron diffraction, which showed a layer-by-layer growth mode. Atomic force microscopy was used to probe the roughness of these samples, which shows very smooth terminations with the surface roughness less than 1 unit cell. The samples were characterized by means of X-ray diffraction data collected at the Rigaku DmaxrB diffractometer, as well as the beamline BL14B1 of Shanghai Synchrotron Radiation Facility, at room temperature. X-ray diffraction results also demonstrate the good quality of supperlattice films. The electronic transport properties and magnetic properties were measured by physical property measurement system, vibrating sample magnetometer, from which the resistance was extracted as a function of temperature ranging from liquid helium temperature to room temperature, shown in Figure 5, as well as average magnetic moment that each Ru owns.

To be restricted, when sample is grown along the crystallographic direction other than [001], surface reconstruction might occur at the substrate surface. For example, Enterkin et al. ${ }^{8}$ has reported surface reconstruction on STO (110) surface. ${ }^{20}$ It affects the physical phenomena at the interface, however, we assume that the contribution from the interface is minor compared with that from the superlattice thin film and thus negligible. The contributions from surface reconstruction, ${ }^{22}$ vacancies, or intermixing should be examined in the following investigation.

\section{ACKNOWLEDGEMENTS}

We acknowledge financial support from NSFC (Nos. U1332205, 11274153, 11204124, 51202108). The authors are also grateful to beamline BL14B in Shanghai Synchrotron Radiation Facility (SSRF) for providing the beam time.

\section{COMPETING INTERESTS}

The authors declare no conflict of interest.

\section{REFERENCES}

1. Li, L., Richter, C., Mannhart, J. \& Ashoori, R. C. Coexistence of magnetic order and two-dimensional superconductivity at $\mathrm{LaAlO}_{3} / \mathrm{SrTiO}_{3}$ interfaces. Nat. Phys. 7, 762 (2011).

2. Michaeli, K., Potter, A. C. \& Lee, P. A. Superconducting and ferromagnetic phases in $\mathrm{SrTiO}_{3} / \mathrm{LaAlO}_{3}$ oxide interface structures: possibility of finite momentum pairing. Phys. Rev. Lett. 108, 117003 (2012).

3. Verissimo-Alves, M., Garćıa-Ferńandez, P., Bilc, D. I., Ghosez, P. \& Junquera, J. Highly confined spin-polarized two-dimensional electron gas in $\mathrm{SrTiO}_{3} / \mathrm{SrRuO}_{3}$ superlattices. Phys. Rev. Lett. 108, 107003 (2012).

4. Gu, M. et al. Magnetic ordering and structural phase transitions in a strained ultrathin $\mathrm{SrRuO}_{3} / \mathrm{SrTiO}_{3}$ superlattice. Phys. Rev. Lett. 109, 157003 (2012).
5. Zayak, A. T., Huang, X., Neaton, J. B. \& Rabe, K. M. Manipulating magnetic properties of $\mathrm{SrRuO}_{3}$ and $\mathrm{CaRuO}_{3}$ with epitaxial and uniaxial strains. Phys. Rev. B 77, 214410 (2008)

6. Ding, H.-C., Li, Y.-W., Zhu, W., Gao, Y.-C. \& Gong, S. J. Improved multiferroic behavior in [111]-oriented $\mathrm{BiFeO}_{3} / \mathrm{BiAlO}_{3}$ superlattice. J. Appl. Phys. 113, 123703 (2013).

7. Kim, T. H. et al. Polar metal by geometric design. Nature 533, 68 (2016).

8. Enterkin, J. A. et al. A homologous series of structures on the surface of $\mathrm{SrTiO}_{3}(110)$. Nat. Mater. 9, 245-248 (2010).

9. Castell, M. R. Scanning tunneling microscopy of reconstructions on the $\mathrm{SrTiO}_{3}$ (001) surface. Surf. Sci. 505, 1-13 (2002).

10. Ohtomo, A. \& Hwang., H. Y. A high-mobility electron gas at the $\mathrm{LaAlO}_{3} / \mathrm{SrTiO}_{3}$ heterointer-face. Nature 427, 423-426 (2004).

11. Herranz, G., Śanchez, F., Dix, N., Scigaj, M. \& Fontcuberta, J. High mobility conduction at (110) and (111) $\mathrm{LaAlO}_{3} / \mathrm{SrTiO}_{3}$ interfaces. Sci. Rep 2, 758 (2012).

12. Annadi, A. et al. Anisotropic two-dimensional electron gas at the $\mathrm{LaAlO}_{3} / \mathrm{SrTiO}_{3}$ (110) interface. Nat. Commun. 4, 1838 (2012).

13. Grutter, A. J., Wong, F. J., Arenholz, E., Vailionis, A. \& Suzuki, Y. Evidence of highspin $\mathrm{Ru}$ and universal magnetic anisotropy in $\mathrm{SrRuO}_{3}$ thin films. Phys. Rev. B 85, 134429 (2012).

14. Kim, B. \& Min, B. I. Termination-dependent electronic and magnetic properties of ultrathin $\mathrm{SrRuO}_{3}$ (111) films on $\mathrm{SrTiO}_{3}$. Phys. Rev. B 89, 195411 (2014).

15. Chang, Y. J., Kim, C. H., Phark, S. H., Kim, Y. S., Yu, J. \& Noh, T. W. Fundamental thickness limit of itinerant ferromagnetic $\mathrm{SrRuO}_{3}$ thin films. Phys. Rev. Lett. 103, 057201 (2009)

16. Rondinelli, J. M., Caffrey, N. M., Sanvito, S. \& Spaldin, N. A. Electronic properties of bulk and thin film $\mathrm{SrRuO}_{3}$ : search for the metal-insulator transition. Phys. Rev. B 78, 155107 (2008)

17. Jeng, H.-T., Lin, S.-H. \& Hsue, C.-S. Orbital ordering and Jahn-Teller distortion in perovskite ruthenate $\mathrm{SrRuO}_{3}$. Phys. Rev. Lett. 97, 067002 (2006).

18. Kresse, G. \& Furthmuller, J. Efficiency of ab-initial total energy calculations for metals and semiconductors using a plane-wave basis set. Comput. Mater. Sci. 6, 15 (1996).

19. Blochl, P. E. Projector augmented-wave method. Phys. Rev. B 50, 17953 (1994).

20. Dudarev, S. L., Botton, G. A., Savrasov, S. Y., Humphreys, C. J. \& Sutton, A. P. Electron-energy-loss spectra and the structural stability of nickel oxide: an LSDA +U study. Phys. Rev. B 57, 1505 (1998).

21. Krukau, A. V., Vydrov, O. A., Izmaylov, A. F. \& Scuseria, G. E. Influence of the exchange screening parameter on the performance of screened hybrid functionals. J. Chem. Phys. 125, 224106 (2006).

22. Mahadevan, P., Aryasetiawan, F., Janotti, A. \& Sasaki, T. Evolution of the electronic structure of a ferromagnetic metal: case of $\mathrm{SrRuO}_{3}$. Phys. Rev. B 80, 035106 (2009).

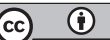

This work is licensed under a Creative Commons Attribution 4.0 International License. The images or other third party material in this article are included in the article's Creative Commons license, unless indicated otherwise in the credit line; if the material is not included under the Creative Commons license, users will need to obtain permission from the license holder to reproduce the material. To view a copy of this license, visit http://creativecommons.org/licenses/ by/4.0/

(c) The Author(s) 2016 